\title{
Placental mesenchymal dysplasia and hepatic cyst
}

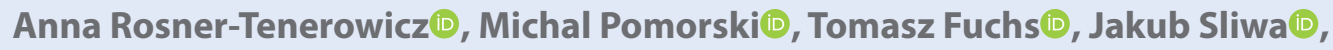 \\ Aleksandra Zimmer-Stelmach $(D$, Wiktor Bek, Mariusz Zimmer(D) \\ $2^{\text {nd }}$ Department of Gynecology and Obstetrics, Wroclaw Medical University, Wroclaw, Poland
}

\begin{abstract}
Placental mesenchymal dysplasia (PMD) is a rare benign vascular anomaly of the placenta. It can be misdiagnosed as a molar pregnancy resulting in unnecessary termination of pregnancy.

A 30-year-old woman was referred to our hospital at 18 gestational weeks due to suspicion of molar pregnancy. The ultrasound showed a bulky placenta with multiple cysts. Oligohydramnion and fetal hypoechogenic cystic area without doppler flow were diagnosed at 23 weeks. The baby was operated on after delivery, and an $80 \mathrm{~mm}$ multifocal cyst originating from the right lobe of the liver was removed. The placenta demonstrated swelling stem villi with enlarged vessels and increased interstitial cells without trophoblast proliferation. PMD and fetal hepatic cyst can coexist; however, the relationship between those conditions remains to be elucidated. PMD is associated with adverse pregnancy outcomes but also with a good prognosis.
\end{abstract}

Key words: placental mesenchymal dysplasia; hepatic cyst

Ginekologia Polska 2020; 91, 12: 779-780

\section{INTRODUCTION}

Placental mesenchymal dysplasia (PMD) is a benign placental, vascular anomaly characterized on ultrasonography by placentomegaly and grape-like vesicles. The incidence of PMD reaches $0.02 \%$ [1].

\section{CASE REPORT}

A 30-year old primipara was referred to hospital at 18 weeks of gestation due to suspicion of molar pregnancy. We diagnosed complete placenta praevia with excessive vascular flow behind the placental bed. The placenta was enlarged, changed by the areas of multiple cystic echoes without vascular flow (Fig. 1 and 2). The ultrasound revealed a normal fetus. The amniotic fluid was diminished. Imaging features raised the possibility of triploidy and partial molar pregnancy. Amniocentesis revealed normal fetal karyotype (46 XX). Polymerase chain reaction analysis of the amniotic fluid for TORCH microorganisms was negative. The patient's blood test revealed an elevated level of AFP. The HCG concentration was normal. The next follow-up at 23 weeks revealed an enlarged placental width with multiple cystic areas and highly vascular retroplacental surface, suggestive of accreta. Additionally, hypoecho-

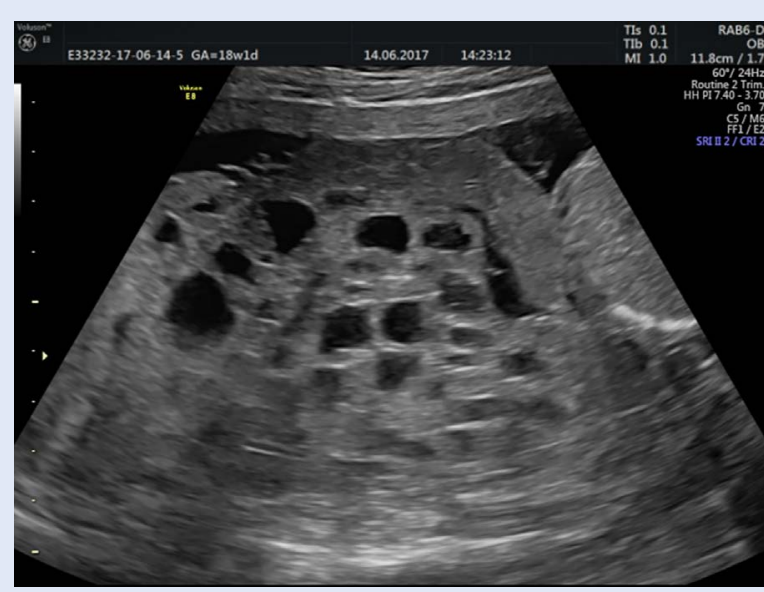

Figure 1. Enlarged placenta with the areas of multiple cystic echoes genic cystic area of $33 \times 21 \mathrm{~mm}$ without any doppler flow was diagnosed in the fetal abdominal cavity. The amount of amniotic fluid was still diminished. At 28 weeks of gestation, the placenta continued to be large and hydropic in ultrasound scanning. The fetal growth curves were normal but the hypoechogenic cyst enlarged to $73 \times 40 \mathrm{~mm}$, changed into multilocules still without any doppler flow. It was located mostly on the right side next to the liver and right kidney (Fig. 3). At 29 weeks 1-day of gestation, the mother presented with pre-term premature rupture of membranes (PROM). Due to pathological CTG, an emergency caesarean delivery was performed. The infant weighed $1320 \mathrm{~g}$ (74 percentile) with Apgar scores of 5 and 6 at 1 and 5 min, respectively. The gasometry from the umbilical artery excluded fetal acidosis. Intraoperatively, the placenta was normally adherent to the uterus. Gross examination of the placenta showed enlarged stem

Corresponding author:

Anna Rosner-Tenerowicz

$2^{\text {nd }}$ Department of Gynecology and Obstetrics, Wroclaw Medical University, 213 Borkowska St, 50-556 Wroclaw, Poland

phone: +48 691 963 950; e-mail: annarosnertenerowicz@gmail.com 


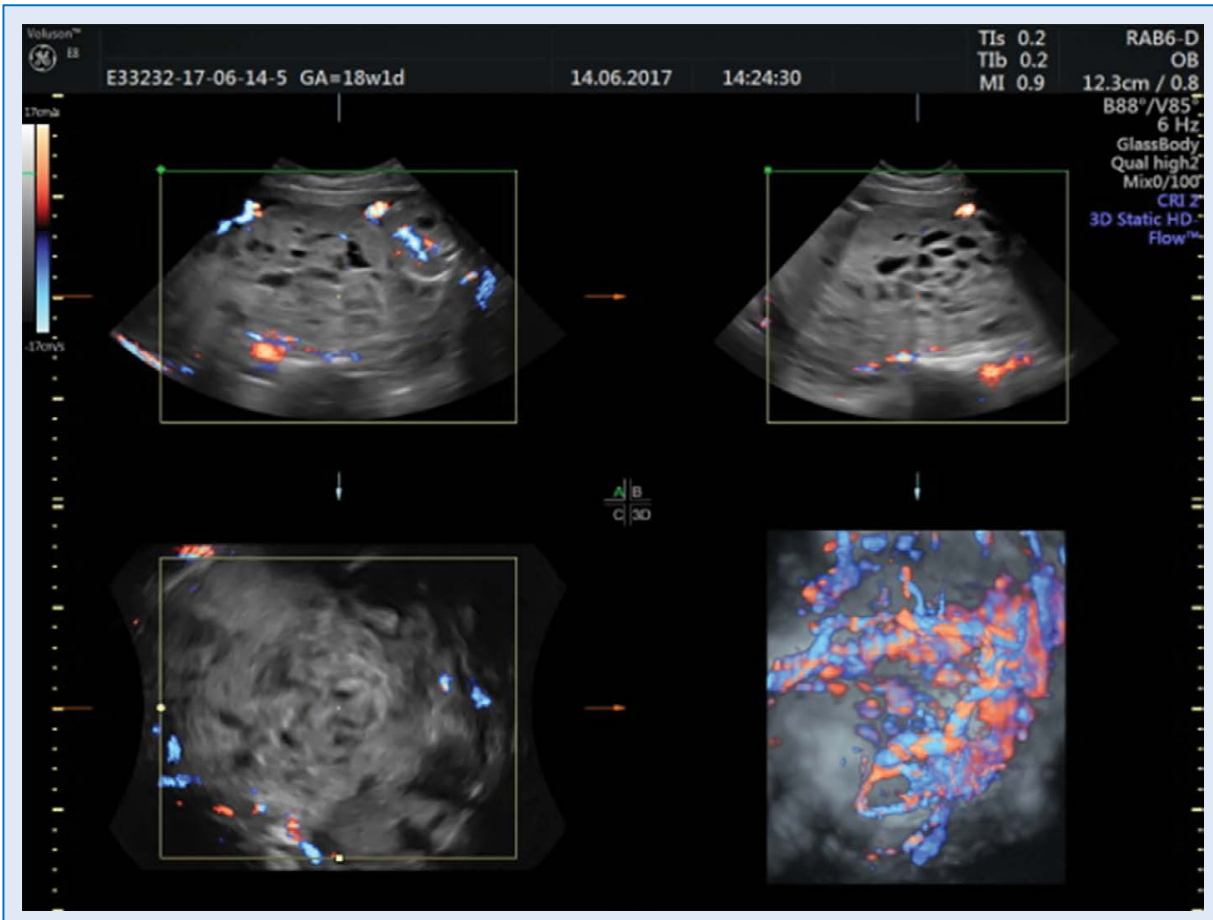

Figure 2. Enlarged placenta with the areas of multiple cystic echoes and excessive vascular flow behind the placental bed

villi. The placenta weighed $500 \mathrm{~g}$ what was around $90^{\text {th }}$ percentile for that age of pregnancy [2]. Microscopic sections showed villi with thick dysplastic vessels surrounded by a myxoid edematous stroma with cistern formation. The findings were consistent with PMD. The neonate did not present with any macroscopic abnormalities at the time of delivery except enlarged abdomen circumference. In postpartum abdominal ultrasound, an $80 \mathrm{~mm}$ multifocal cyst was confirmed. The neonate was operated on day 4 post-delivery. The cyst appeared to have the origin in the right lobe of the liver. The outcome of the

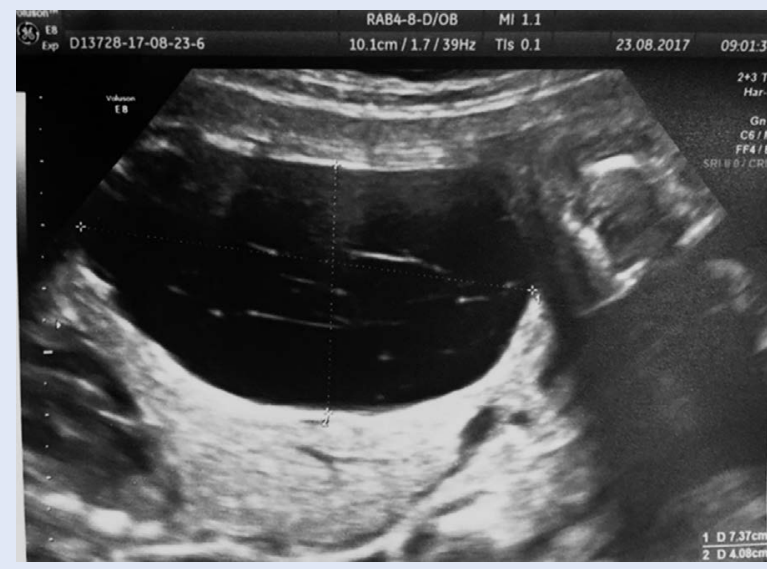
histopathological examination was the simple cyst of the liver. The baby was discharged at seven weeks after caesarean section in a good condition. The mother recovered normally and was discharged home on day 4 of postpartum.

\section{DISCUSSION}

In literature cases with PMD revealed cystic placentas in $80 \%$, enlarged in $50 \%$ and dilated chorionic vessels in $16 \%$ [3]. Increased hCG level was found in $38 \%$ and AFP in $70 \%$ of cases [3]. The most common complication was preterm delivery (52\%), PROM reached $17 \%$. Intrauterine growth restriction (IUGR) occurred in $33 \%$. Genetic abnormalities were found in $28 \%$, Beckwith-Wiedemann syndrome was the most common, reaching $23 \%$ [4]. Neonatal hepatic tumors were diagnosed in $17 \%$ of PMD, mostly hepatic mesenchymal tumors [3]. Intrauterine fetal

Figure 3. Hypoechogenic cyst in fetal abdominal cavity death was reported in $13 \%$ of cases, but $9 \%$ of patients had normal pregnancy outcomes [3]. It is important to distinguish PMD
from a partial mole because incorrect diagnosis may result in pregnancy termination. PMD is associated with adverse pregnancy outcomes but also with a good prognosis.

\section{REFERENCES}

1. Arizawa M, Nakayama M. Suspected involvement of the X chromosome in placental mesenchymal dysplasia. Congenit Anom (Kyoto). 2002; 42(4): 309-317, doi: 10.1111/j.1741-4520.2002.tb00897.x, indexed in Pubmed: 12634450.

2. Thompson JMD, Irgens LM, Skjaerven R, et al. Placenta weight percentile curves for singleton deliveries. BJOG. 2007; 114(6): 715-720, doi: 10.1111/j.1471-0528.2007.01327.x, indexed in Pubmed: 17516963.

3. Nayeri UA, West AB, Grossetta Nardini HK, et al. Systematic review of sonographic findings of placental mesenchymal dysplasia and subsequent pregnancy outcome. Ultrasound Obstet Gynecol. 2013; 41(4): 366-374, doi: 10.1002/uog.12359, indexed in Pubmed: 23239538.

4. H'mida D, Gribaa M, Yacoubi T, et al. Placental mesenchymal dysplasia with beckwith-wiedemann syndrome fetus in the context of biparental and androgenic cell lines. Placenta. 2008; 29(5): 454-460, doi: 10.1016/j.placenta.2008.01.001, indexed in Pubmed: 18342934. 